\title{
OPTIMAL TRAJECTORIES ASSOCIATED TO A SOLUTION OF CONTINGENT HAMILTON-JACOBI EQUATION
}

\author{
Halina Frankowska*
}

June 1987

WP-87-069

${ }^{*}$ CEREMADE, Université PARIS-IX Dauphine, Paris, Cedex 16, France

Working Papers are interim reports on work of the International Institute for Applied Systems Analysis and have received only limited review. Views or opinions expressed herein do not necessarily represent those of the Institute or of its National Member Organizations.

INTERNATIONAL INSTITUTE FOR APPLIED SYSTEMS ANALYSIS A-2361 Laxenburg, Austria 


\title{
Foreword
}

Every smooth solution of a Hamilton-Jacobi-Bellman equation in optimal control (if it does exist) can be used for testing the optimality trajectories of a control system and to construct an optimal feedback. Since, in general, such a smooth solution does not exist, one has to deal with less regular solutions, for example with viscosity solutions. In this paper the author describes a subclass of viscosity solutions which on one hand can be used for the construction of optimal feedback and on the other provides a sufficient condition for optimality.

\author{
Alexander B. Kurzhanski \\ Chairman \\ System and Decision Sciences Program
}




\begin{abstract}
In this paper we study the existence of optimal trajectories associated with a generalized solution to Hamilton-Jacobi-Bellman equation arising in optimal control.

In general, we cannot expect such solutions to be differentiable. But, in a way analogous to the use of distributions in PDE, we replace the usual derivatives with "contingent epiderivatives" and the Hamilton-Jacobi equation by two "contingent Hamilton-Jacobi inequalities".
\end{abstract}

We show that the value function of an optimal control problem verifies these "contingent inequalities".

Our approach allows the following three results:

(a) The upper semicontinuous solutions to contingent inequalities are monotone along the trajectories of the dynamical system.

(b) With every continuous solution $V$ of the contingent inequalities, we can associate an optimal trajectory along which $V$ is constant.

(c) For such solutions, we can construct optimal trajectories through the corresponding optimal feedback.

They are also "viscosity solutions" of a Hamilton-Jacobi equation. Finally we prove a relationship between superdifferentials of solutions introduced in Crandall-Evans-Lions [10] and the Pontrjagin principle and discuss the link of viscosity solutions with Clarke's approach to the Hamilton-Jacobi equation. 


\title{
OPTIMAL TRAJECTORIES ASSOCIATED \\ TO A SOLUTION OF CONTINGENT \\ HAMILTON-JACOBI EQUATION
}

\author{
Halina Frankowska
}

\section{Introduction}

Consider the problem

$$
\text { minimize } g(x(1))
$$

over the solutions to the control system

$$
\begin{aligned}
& x^{\prime}=f(t, x, u(t)), \quad u(t) \in U \\
& x(0)=x_{0}
\end{aligned}
$$

where $g$ is a function taking values in the extended real line $R \cup\{+\infty\}$. We recall that a problem with terminal constraints, $x(1) \in C$, can be rewritten in the above form by setting $g(x)=+\infty$ whenever $x \notin C$.

The dynamic programming approach associates with the above problem the "value function" defined by: for all $(t, \xi) \in[0,1] \times \mathbf{R}^{n}$

$$
V(t, \xi)=\inf \{g(x(1)): x \text { is a solution of }(2) \text { on }[t, 1], x(t)=\xi\}
$$

(see $[4],[5],[22])$.

In the case when the value function is differentiable, it satisfies the Hamilton-Jacobi equation

$$
\begin{aligned}
& \frac{\partial}{\partial t} V(t, x)+\inf _{u \in U} \frac{\partial}{\partial x} V(t, x) f(t, x, u)=0 \\
& V(1, x)=g(x)
\end{aligned}
$$

where $\frac{\partial}{\partial t} V, \frac{\partial}{\partial x} V$ denote the derivatives of $V$ with respect to $t$ and $x$ respectively. 
Although the value function fails to be continuously differentiable even when the data are smooth, the equations (4), (5) still have an important feature: if $V$ is a $C^{1}$ solution of the equation (4) satisfying the boundary condition (5), the "verification technique" amounts to recognize an optimal trajectory-control pair $\left(x_{*}, u_{*}\right)$ of system (2), (3) by checking that for almost all $t \in[0,1]$

$$
\frac{\partial}{\partial t} V(t, x *(t))+\frac{\partial}{\partial x} V(t, x *(t)) f(t, x *(t), u *(t))=0
$$

In general the nonlinear boundary problem (4), (5) does not have a global continuously differentiable solution even if $f$ and $g$ are smooth.

In this paper we show that the value function $V$ is a solution of a boundary problem involving "contingent inequalities".

The contingent derivative $D_{+} \varphi\left(x_{0}\right)$ of a function $\varphi: \mathbf{R}^{m} \rightarrow \mathbf{R} \cup\{ \pm \infty\}$ is defined at every point $x_{0}$ where $\varphi\left(x_{0}\right)$ is finite by: for all $u \in \mathbf{R}^{m}$

$$
D_{+} \varphi\left(x_{0}\right) u=\lim _{\substack{h \rightarrow 0+\\ u \rightarrow u}} \inf \left[\varphi\left(x_{0}+h u^{\prime}\right)-\varphi\left(x_{0}\right)\right] / h
$$

We prove that the value function verifies on $\left[0,1\left[\times \mathbf{R}^{n}\right.\right.$ the following contingent inequalities:

$$
\begin{gathered}
\sup _{u \in U} D_{+}(-V)(t, x)(1, f(t, x, u)) \leq 0 \\
\inf _{u \in U} D_{+} V(t, x)(1, f(t, x, u)) \leq 0
\end{gathered}
$$

and the boundary condition

$$
V(1, x)=g(x) \text { on } \mathbf{R}^{n}
$$

When $V$ is differentiable at $(t, x)$, the inequalities $(7),(8)$ reduce to equation (4). We shall observe that the value function is smaller than any function continuous on its domain, satisfying relations (7) - (9).

Inequalities (7) and (8) have two different tasks to perform:

(a) Every upper semicontinuous solution $V$ of the inequality (7) is nondecreasing along the trajectories of dynamical system (2).

(b) For control systems with convex right-hand side, to every continuous solution $V$ of inequality (8) corresponds a trajectory $z$ of (2), (3) such that $V$ is nonincreasing along $z$. 
This means that in the case when the sets $f(t, x, U)$ are convex, to every continuous solution $V$ of (7)-(9) corresponds at least one optimal trajectory $z$ of problem (1)-(3) such that $V$ is constant along $z$. When the value function is locally Lipschitzian then it verifies the equation

$$
\inf _{u \in U} D_{+} V(t, x)(1, f(t, x, u))=0, \quad t \in\left[0,1\left[\times \mathbf{R}^{n}\right.\right.
$$

instead of inequality (8). Obviously the solutions to (10) have the property (b) mentioned above.

On the other hand any locally Lipschitzian solution to the boundary problem

$$
\left.\begin{array}{l}
\inf _{u \in U} D_{+} V(t, x)(1, f(t, x, u)) \leq 0, \quad(t, x) \in\left[0,1\left[\times \mathbf{R}^{n}\right.\right. \\
\sup _{u \in U} D_{+}(-V)(t, x)(1, f(t, x, u)) \leq 0, \quad(t, x) \in\left[0,1\left[\times \mathbf{R}^{n}\right.\right. \\
V(1, x)=g(x)
\end{array}\right\}
$$

provides a test for optimality analogous to (6). Namely if $V$ is a locally Lipschitzian solution to the boundary problem (11) then any trajectory-control pair $(x *, u *)$ of $(2),(3)$ verifying for almost all $t \in[0,1]$

$$
D_{+} V(t, x *(t))(1, f(t, x *(t), u *(t))=0
$$

is optimal for problem (1)-(3). This implies in particular that every solution defined on time interval $[0,1]$ of the closed loop control system

$$
x^{\prime}=f(t, x, u), u \in W(t, x), \quad x(0)=x_{0}
$$

where $W(t, x)=\left\{u \in U: D_{+} V(t, x)(1, f(t, x, u))=0\right\}$ is optimal for problem (1)-(3).

We also check that every solution $V$ of boundary problem (7)-(9) is a viscosity solution in the sense of Crandall-Evans-Lions [9], [10] of the Hamilton-Jacobi-Bellman equation:

$$
\begin{aligned}
& -\frac{\partial}{\partial t} V+H\left(t, x,-\frac{\partial}{\partial x} V\right)=0 \text { on }\left[0,1\left[\times \mathbf{R}^{n}\right.\right. \\
& V(1, x)=g(x) \text { on } \mathbf{R}^{n}
\end{aligned}
$$

where the Hamiltonian $H$ is defined by

$$
H(t, x, p)=\sup _{u \in U}\langle p, f(t, x, u)>
$$

The converse statement is not generally true. 
It is not known yet, at least to our knowledge, whether we can recover optimal trajectories or optimal feedbacks from viscosity solutions to the Hamilton-Jacobi-Bellman equation of an optimal control problem. It is still possible whenever a viscosity solution is the value function, or, more generally whenever a viscosity solution verifies in addition inequalities (8), (9).

The locally Lipschitzian value function satisfies also an extension of Hamilton- Jacobi equation introduced in Offin [21] (see Clarke [8], Clarke-Vinter [6]).

$$
\min _{(p, q) \in \partial V(t, x)}\left\{p+\min _{u \in U}<q, f(t, x, u)>\right\}=0
$$

where $\partial V$ denotes the generalized gradient of $V$. Any Lipschitzian solution of (16) is nondecreasing along trajectories of dynamical system (2). This allows to prove a sufficient condition for optimality for a given trajectory (see Clarke-Vinter [6]). However it does not mean that with every solution of (16), (9) we can relate an optimal trajectory of problem (1)-(3) as the relation (6) does.

A locally Lipschitzian value function verifies not only equation (16) but also equation

$$
\max _{\pi_{t} \partial V(t, x)} p=\max _{q \in \pi_{x} \partial V(t, x)} H(t, x,-q)
$$

where $\pi_{t}$ and $\pi_{x}$ denote the corresponding projections.

The outline of the paper is as follows. In Section 1 we state the basic hypotheses and prove some preliminary results. In Section 2 we show that the value function verifies contingent inequalities (7)-(10) and that continuous solutions of (7)-(9) are viscosity solutions of (14). Section 3 is devoted to properties of solutions of contingent inequalities and Section 4 to the optimal feedback. In Section 5 we prove some other relations verified by the value function and provide a short proof of (16). Finally in the last section we study the relationship between the superdifferentials introduced in [10] and the adjoint vector of the Pontrjagin maximum principle.

\section{Basic assumptions and preliminary results}

In this paper we consider a dynamical system described by a differential inclusion.

Let $F$ be a set-valued map from $[0,1] \times \mathbf{R}^{n}$ to $\mathbf{R}^{n}$. We associate with it the differential inclusion

$$
x \in F(t, x)
$$


A function $x \in W^{1,1}(t, T), T \geq t$ (the Sobolev space) is called a trajectory of the differential inclusion (1.1) if for almost all $s \in[t, T], x^{\prime}(s) \in F(s, x(s))$. The set of all trajectories of (1.1) defined on the time interval $[t, T]$ and starting at $\xi,(x(t)=\xi)$ is denoted by $S_{[t, T]}(\xi)$

Let $B$ be the closed unit ball in $\mathbf{R}^{n}$. Throughout the whole paper we assume that for all $(t, x) \in[0,1] \times \mathbf{R}^{n}$

$\left(H_{1}\right) F(t, x)$ is a nonempty compact set,

that for all $x \in \mathbf{R}^{n}$

$\left(H_{2}\right) \quad F(\cdot, x)$ is continuous on $[0,1]$

and

$\left(H_{3}\right) \quad F$ is locally Lipschitzian in $x$, in the sense that for every $\left(t_{0}, x_{0}\right) \in[0,1] \times \mathbf{R}^{n}$ there exists a neighborhood $\mathbf{N}$ in $[0,1] \times \mathbf{R}^{n}$ and a constant $L$ such that for all $(t, x),(t, y) \in \mathbf{N}$

$$
F(t, x) \subset F(t, y)+L\|x-y\| B
$$

Example. Consider the closed loop control system

$$
\begin{aligned}
& x^{\prime}(t)=f(t, x(t), u(t)) \\
& u(t) \in U(x(t))
\end{aligned}
$$

where $f:[0,1] \times \mathbf{R}^{n} \times \mathbf{R}^{m} \rightarrow \mathbf{R}^{n}$ is a continuous function and $U: \mathbf{R}^{n} \rightrightarrows \mathbf{R}^{m}$ is a continuous control map with nonempty compact images. Admissible controls are measurable functions on $[0,1]$ satisfying (1.4).

For all $(t, x) \in \mathbf{R} \times \mathbf{R}^{n}$ set

$$
F(t, x)=\{f(t, x, u): u \in U(x)\}
$$

Clearly every trajectory of (1.3), (1.4) is a trajectory of the differential inclusion (1.1) with $F$ defined as in (1.5). Conversely, with every trajectory $x \in S_{[0 ; 1]}$ of differential inclusion (1.1) we can associate a measurable selection $u(t) \in U(x(t))$ such that (1.3) holds true almost everywhere in $[0,1]$. This follows from Lusin's theorem exactly by the same arguments as in [1, p. 91] (see also [8, pp. 111-112]).

Hence we can rewrite the dynamical system (1.3), (1.4) in the differential inclusion formulation (1.1) with $F$ defined by (1.5).

The set-valued map $F$ satisfies hypothesis $\left(H_{1}\right)$ and $\left(H_{2}\right)$. If moreover $U$ is locally Lipschitzian and $f$ is locally Lipschitzian in $(x, u)$, so is $F$. 
For all $t \in \mathbf{R}, T \geq t$ and $\xi \in \mathbf{R}^{n}$ set

$$
R(T, t) \xi=\left\{x(T): x \in S_{[t, T]}(\xi)\right\}
$$

This is the so-called reachable set of $(1.1)$ from $(t, \xi)$ at time $T$.

When $F$ is sufficiently regular the set $\operatorname{co} F(t, \xi)$ is the infinitesimal generator of the semigroup $R(\cdot, t) \xi$ (see Frankowska [15]).

The following theorem provides a more precise result concerning reachable sets.

Theorem 1.1. Assume that the assumptions $\left(H_{1}\right)-\left(H_{3}\right)$ are verified. Then for every $\left(t_{0}, \xi_{0}\right) \in\left[0,1\left[\times \mathbf{R}^{n}\right.\right.$ and all $(t, \xi)$ near $\left(t_{0}, \xi_{0}\right)$ and small $h>0$

$$
R(t+h, t) \xi=\xi+h \text { co } F\left(t_{0}, \xi_{0}\right)+0(t, \xi, h)
$$

where

$$
\lim _{\substack{(t, \xi) \rightarrow\left(t_{0}, \xi_{0}\right) \\ h \rightarrow 0+}}\|0(t, \xi, h)\| / h=0
$$

Remark. Equality (1.7) means that

$$
\begin{aligned}
& R(t+h, t) \xi \subset \xi+h \text { co } F\left(t_{0}, \xi_{0}\right)+\|0(t, \xi, h)\| B \text { and } \\
& \xi+h \operatorname{co} F\left(t_{0}, \xi_{0}\right) \subset R(t+h, t) \xi+\|0(t, \xi, h)\| B
\end{aligned}
$$

Proof. Let $\rho>0$ be such that $F(t, \cdot)$ is $L$-Lipschitzian on $\left[t_{0}-\rho, t_{0}+\rho\right] \times\left(\xi_{0}+\rho B\right)$, where $L$ denotes the Lipschitz constant of $F$ with respect to $x$. Since $F$ is continuous and has compact images there exists $M \geq 1$ such that for all $\left|t-t_{0}\right| \leq \rho, \xi \in \xi_{0}+\rho B$ we have $F(t, \xi) \subset M B$.

Define

$$
\mathbf{N}=\left\{(t, \xi):\left|t-t_{0}\right| \leq p / 2,\left\|\xi-\xi_{0}\right\| \leq p / 2\right\}
$$

and observe that for all $T \geq t,\left\|\xi-\xi_{0}\right\| \leq \rho / 2$ and $x \in S_{[t, T]}(\xi)$ verifying $\left.x(\mid t, T]\right) \subset \xi_{0}+\rho B$, $\left\|x(T)-\xi_{0}\right\| \leq\|x(T)-\xi\|+\rho / 2 \leq \int_{t}^{T}\left\|x^{\prime}(s)\right\| d s+\rho / 2 \leq M(T-t)+\rho / 2$. Hence for all $h \in[0, \rho / 2 M]$ and $(t, \xi) \in \mathbf{N}$ the set $S_{[t, t+h]}(\xi) \neq \varnothing$ and for every $x \in S_{[t, t+h]}(\xi)$ and $s \in[t, t+h]$

$$
\|x(s)-\xi\| \leq M(s-t)
$$

Since $F$ is uniformly continuous on the compact set $\left\{(t, \xi):\left|t-t_{0}\right| \leq \rho,\left\|\xi-\xi_{0}\right\| \leq \rho\right\}$ for every $\epsilon>0$ there exists $\delta>0$ such that 


$$
F(t+h, x) \subset F(t, \xi)+\epsilon B \text { for all }(t, \xi) \in \mathbf{N}, h \in[0, \delta] \text { and } x \in \xi+\delta B
$$

Using (1.8) and the mean value theorem $[1$, p. 21] we obtain that for all $0<h \leq \min \{\delta / M, \rho / 2 M\},(t, \xi) \in \mathrm{N}$ and $x \in S_{[t, t+h]}(\xi)$

$$
x(t+h)=\xi+\int_{t}^{t+h} x(s) d s \in \xi+\int_{t}^{t+h} F(s, x(s)) d s \subset \xi+\int_{t}^{t+h}(F(t, \xi)+\epsilon B) d s=\xi+h \text { co } F(t, \xi)+\epsilon h B
$$

This implies that

$$
R(t+h, t) \xi \subset \xi+h \text { co } F(t, \xi)+\epsilon h B
$$

for all $(t, \xi) \in \mathbf{N}$ and sufficiently small $h>0$. Since $\epsilon>0$ is arbitrary and $F$ is continuous at $\left(t_{0}, \xi_{0}\right)$ we proved that for all $(t, \xi)$ near $\left(t_{0}, \xi_{0}\right)$ and small $h>0$

$$
R(t+h, t) \xi \subset \xi+h \text { co } F\left(t_{0}, \xi_{0}\right)+\|0(t, \xi, h)\| B
$$

To prove the opposite inclusion observe that by the relaxation theorem $[1$, p. 124] reachable sets of (1.1) are dense in the reachable sets of the convexified inclusion. Thus we may assume that $F$ has convex images. For all $(t, \xi) \in \mathrm{N}$ and $u \in F(t, \xi)$ let $u_{t, \xi}(s)$ denote the projection of $u$ on $F(t+s, \xi)$. Since $F$ is continuous on $N\left\{u_{t, \xi}\right\}_{(t, \xi) \in N}$ is a family of equicontinuous functions on $[0, \rho / 2]$. Hence for all $\epsilon>0$ there exists $\delta>0$ such that for all $s \in[0, \delta],\left\|u_{t, \xi}(s)-u_{t, \xi}(0)\right\| \leq \epsilon$. Therefore for all $(t, \xi) \in \mathbf{N}, u \in F(t, \xi)$ and $h \in[0, \delta]$

$$
\left\|\int_{0}^{h} u_{t, \xi}(\tau) d \tau-h u\right\| \leq \epsilon h
$$

On the other hand for all $s \in[0, \rho / 2 M]$,

$$
\operatorname{dist}\left(u_{t, \xi}(s), F\left(t+s, \xi+\int_{0}^{s} u_{t, \xi}(\tau) d \tau\right)\right) \leq L \int_{0}^{s}\left\|u_{t, \xi}(\tau)\right\| d \tau
$$

By $\left[1\right.$, p. 120] there exist $\delta_{1}>0, M_{1}>0$ which depend only on $L$ and $M$ such that for all $h \in\left[0, \delta_{1}\right]$

$$
\operatorname{dist}\left(\xi+\int_{0}^{h} u_{t, \xi}(\tau) d \tau, R(t+h, t) \xi\right) \leq M_{1} \int_{0}^{h} \int_{0}^{s}\left\|u_{t, \xi}(\tau)\right\| d \tau d s
$$

Hence, by (1.9), for all $0 \leq h \leq \min \left\{\delta, \delta_{1}, \rho / 2 M\right\},(t, \xi) \in \mathbf{N}$ and $u \in F(t, \xi)$

$$
\operatorname{dist}(\xi+h u, R(t+h, t) \xi) \leq \epsilon h+M_{1} \int_{0}^{h} \int_{0}^{s}\left\|u_{t, \xi}(\tau)\right\| d \tau d s \leq \epsilon h+M_{1} M h^{2}
$$


Since $M_{1}$ does not depend on $\epsilon$ and $\delta, \delta_{1}$ do not depend on $(t, \xi) \in \mathbf{N}$ and $u \in F(t, \xi)$ we finally obtain that for all $(t, \xi)$ near $\left(t_{0}, \xi_{0}\right)$ and small $h>0$

$$
\xi+h \operatorname{co} F\left(t_{0}, \xi_{0}\right) \subset R(t+h, t) \xi+\|0(t, \xi, h)\| B
$$

Thus

$$
R(t+h, t) \xi=\xi+h \operatorname{co} F\left(t_{0}, \xi_{0}\right)+0(t, \xi, h)
$$

\section{Contingent inequalities for value function}

Consider the problem

$$
\operatorname{minimize}\left\{g(x(1)): x \in S_{[0,1]}\left(x_{0}\right)\right\}
$$

where $g$ is a function from $\mathbf{R}^{n}$ to the extended real line $\mathbf{R} \cup\{+\infty\}$ and $S_{[0,1]}$ has the same meaning as in Section 1.

The value function associated to this problem is defined by: for all $t \in[0,1], \xi \in \mathbf{R}^{n}$

$$
V(t, \xi)=\inf \left\{g(x(1)): x \in S_{[t, 1]}(\xi)\right\}
$$

If $S_{\mid t, 1]}(\xi)=\varnothing$ then we set $V(t, \xi)=+\infty$.

The following properties of $V$ are an immediate consequence of the definition of a value function (compare $[4],[5],[20])$ :

$$
\begin{gathered}
\qquad(1, x)=g(x) \text { for all } x \in \mathbf{R}^{n} \\
\forall x \in S_{[t, 1]} \text { the function }[t, 1] \ni s \rightarrow V(s, x(s)) \text { is nondecreasing }
\end{gathered}
$$

Moreover if $z \in S_{[0,1]}\left(x_{0}\right)$ solves the problem (2.1) then

$$
V(s, z(s)) \equiv g(z(1)) \text { on }[0,1] .
$$

Conversely we have

Proposition 2.1. If a function $V:[0,1] \times \mathbf{R}^{n} \rightarrow \mathbf{R} \bigcup\{ \pm \infty\}$ and a trajectory $z \in S_{[0,1]}\left(x_{0}\right)$ satisfy (2.3)-(2.5) then $z$ is an optimal solution of the problem (2.1).

The proof follows from the classical arguments of [5, p.89], [12, p.82] and [22, p.87].

Definition 2.2 (contingent derivative). Let $X$ be a subset of $\mathbf{R}^{m}, \varphi: X \rightarrow \mathbf{R} \cup\{ \pm \infty\}$ be a given function and $x_{0} \in X$ be such that $\varphi\left(x_{0}\right) \neq \pm \infty$. The contingent derivative of $\varphi$ at $x_{0}$ is the function $D_{+} \varphi\left(x_{0}\right): \mathbf{R}^{m} \rightarrow \mathbf{R} \cup\{ \pm \infty\}$ defined by: for all $\boldsymbol{u} \in \mathbf{R}^{m}$ 


$$
D_{+} \varphi\left(x_{0}\right) u=\liminf _{\substack{\left(u^{\circ}, h\right) \rightarrow(u, 0+) \\ x_{0}+h \varepsilon \in X}}\left[\varphi\left(x_{0}+h u^{\prime}\right)-\varphi\left(x_{0}\right)\right] / h
$$

The epigraph of $D_{+} \varphi\left(x_{0}\right)$ is equal to the contingent cone to the epigraph of $\varphi$ at $\left(x_{0}, \varphi\left(x_{0}\right)\right)$. If for all $u \in \mathbf{R}^{m}, D_{+} \varphi\left(x_{0}\right) u>-\infty$ then $D_{+} \varphi\left(x_{0}\right)$ is positively homogeneous and lower semicontinuous (see [2, Chapter 7]).

Recall that the domain of definition of $V$ is given by

$$
\operatorname{dom} V:=\{(t, x): V(t, x) \neq \pm \infty\}
$$

and let $V_{d}$ denote the restriction of $V$ to the set dom $V$.

Theorem 2.3. The value function $V$ satisfies the following inequalities:

$$
\begin{aligned}
& \inf _{u \in \operatorname{coF}(t, x)} D_{+} V_{d}(t, x)(1, u) \leq 0 \\
& \sup _{u \in \operatorname{cof}(t, x)} D_{+}(-V)(t, x)(1, u) \leq 0,(t, x) \in \operatorname{Dom} V, \quad t<1
\end{aligned}
$$

If moreover for some $t \in[0,1[, V(t, \cdot)$ is locally Lipschitz at $x$ then

$$
\min _{u \in c o F(t, x)} D_{+} V(t, x)(1, u)=0
$$

Proof. Fix $(t, x) \in \operatorname{Dom} V, t<1$. Then for all small $h>0$, $V(t, x)=\inf \{V(t+h, w): w \in R(t+h, t) x\}$. Hence for some $w_{h} \in R(t+h, t) x$

$$
\lim _{h \rightarrow 0+}\left[V\left(t+h, w_{h}\right)-V(t, x)\right] / h=0
$$

By Theorem 1.1 for all $h>0, w_{h}=x+h u_{h}$ where $\lim _{h \rightarrow 0+} \operatorname{dist}\left(u_{h}, F(t, x)\right)=0$. Let $h_{i} \rightarrow 0+$ and $u_{i}=u_{h_{i}}$ be such that $\lim _{i \rightarrow \infty} u_{i}=\bar{u} \in F(t, x)$. From (2.9) we obtain

$$
\lim _{i \rightarrow \infty}\left[V_{d}\left(t+h_{i}, x+h_{i} u_{i}\right)-V_{d}(t, x)\right] / h_{i}=0
$$

Hence $D_{+} V_{d}(t, x)(1, \bar{u}) \leq 0$ and (2.6) follows. To prove (2.7) observe that for all $w \in R(t+h, t) x, V(t, x) \leq V(t+h, w)$. Fix $\bar{u} \in c o F(t, x)$. By Theorem 1.1 there exist $u_{h} \rightarrow \bar{u}$ such that for all $h>0, x+h u_{h} \in R(t+h, t) x$. Hence $D_{+}(-V)(t, x)(1, \bar{u}) \leq \limsup _{h \rightarrow 0+}\left[-V\left(t+h, x+h u_{h}\right)+V(t, x)\right] / h \leq 0$. Since $\bar{u}$ is arbitrary we proved (2.7). Let $(t, x) \in\left[0,1\left[\times \mathbf{R}^{n}\right.\right.$ be such that $V(t, \cdot)$ is locally Lipschitzian at $x$. Then, by $[2, \mathrm{p} .418], D_{+} V(t, x)$ is lower semicontinuous. Since co $F(t, x)$ is compact there exists $\bar{u} \in$ co $F(t, x)$ such that $D_{+} V(t, x)(1, \bar{u})=\inf _{u \in c o F(t, x)} D_{+} V(t, x)(1, u)$. By Theorem 1.1 for 
some $u_{h} \rightarrow \bar{u}, \quad x+h u_{h} \in R(t+h, t) x$. Thus $V\left(t+h, x+h u_{h}\right)-V(t, x) \geq 0$ and by the local Lipschitzianity of $V, D_{+} V(t, x)(1, \bar{u}) \geq 0$. Hence (2.6) implies (2.8).

Inequalities (2.6), (2.7) can be considered as an extension of Hamilton-Jacobi equation because of the following

Corollary 2.4. If a function $V$ satisfying (2.6) and (2.7) is differentiable at $(t, x)$ then

$$
\frac{\partial}{\partial t} V(t, x)+\inf _{u \in F(t, x)} \frac{\partial}{\partial x} V(t, x) u=0
$$

Proof. Fix $(t, x)$ as above. Then for all $u \in F(t, x)$,

$$
D_{+} V(t, x)(1, u)=\frac{\partial}{\partial t} V(t, x)+\frac{\partial}{\partial x} V(t, x) u
$$

and

$$
D_{+}(-V)(t, x)(1, u)=-\frac{\partial}{\partial t} V(t, x)-\frac{\partial}{\partial x} V(t, x) u
$$

Moreover

$$
\inf _{u \in c o F(t, x)} \frac{\partial}{\partial x} V(t, x) u=\inf _{u \in F(t, x)} \frac{\partial}{\partial x} V(t, x) u
$$

Thus (2.11) is a consequence of (2.6), (2.7). $\square$

Define the Hamiltonian $H$ by: for all $(t, x) \in[0,1] \times \mathbf{R}^{n}$ and $q \in \mathbf{R}^{n}$

$$
H(t, x, q)=\sup \{\langle q, e>: e \in F(t, x)\}
$$

Our next aim is to show that for any open set $\Omega \subset[0,1] \times \mathbf{R}^{n}$ every solution of the problem

$$
\left.\begin{array}{c}
\inf _{u \in \operatorname{co} F(t, x)} D_{+} V(t, x)(1, u) \leq 0,(t, x) \in \Omega \\
\sup _{u \in c o F(t, x)} D_{+}(-V)(t, x)(1, u) \leq 0,(t, x) \in \Omega
\end{array}\right\}
$$

is the viscosity solution of Hamilton-Jacobi equation

$$
-\frac{\partial}{\partial t} V+H\left(t, x,-\frac{\partial}{\partial x} V\right)=0,(t, x) \in \Omega
$$

(see Crandall-Lions [9], and Crandall-Evans-Lions [10]). Some related results can be found in $[20]$. We recall first 
Definition 2.5 (super- and subdifferentials). Let $\Omega \subset \mathbf{R}^{m}$ be an open set, $\varphi$ be a function from $\Omega$ to $\mathbf{R}$ and $x_{0} \in \Omega$.

The superdifferential of $\varphi$ at $x_{0}$ is the set

$$
\partial_{+} \varphi\left(x_{0}\right)=\left\{p: \limsup _{x \rightarrow x_{0}}\left[\varphi(x)-\varphi\left(x_{0}\right)-<p, x-x_{0}>\right] /\left\|x-x_{0}\right\| \leq 0\right\}
$$

The subdifferential of $\varphi$ at $x_{0}$ is the set

$$
\partial_{-} \varphi\left(x_{0}\right)=\left\{p: \liminf _{x \rightarrow x_{0}}\left[\varphi(x)-\varphi\left(x_{0}\right)-<p, x-x_{0}>\right] /\left\|x-x_{0}\right\| \geq 0\right\} .
$$

The super and subdifferentials are closed, possibly empty, convex sets.

Definition 2.6 (viscosity solution). A function $V: \Omega \rightarrow \mathbf{R}$ is called a viscosity solution of the equation (2.14) if for every $(t, x) \in \Omega$ we have

a) for all $p=\left(p_{0}, \ldots, p_{n}\right) \in \partial_{+} V(t, x)$

$$
-p_{0}+H\left(t, x,-\left(p_{1}, \ldots, p_{n}\right)\right) \leq 0
$$

(viscosity subsolution).

b) for all $p=\left(p_{0}, \ldots, p_{n}\right) \in \partial_{-} V(t, x)$

$$
-p_{0}+H\left(t, x,-\left(p_{1}, \ldots, p_{n}\right)\right) \geq 0 \text {. }
$$

(viscosity supersolution).

Lemma 2.7. Let $\Omega$ be an open set and $\varphi: \Omega \rightarrow \mathbf{R}$. Then

$$
\begin{aligned}
& \partial_{-} \varphi\left(x_{0}\right)=\left\{p: \forall r \in \mathbf{R}^{m}, D_{+} \varphi\left(x_{0}\right) r \geq<p, r>\right\} \\
& \partial_{+} \varphi\left(x_{0}\right)=\left\{p: \forall r \in \mathbf{R}^{m}, D_{+}(-\varphi)\left(x_{0}\right) r \geq<-p, r>\right\}
\end{aligned}
$$

Proof. Fix $p \in \partial_{-} \varphi\left(x_{0}\right)$ and $r \in \mathbf{R}^{m}$. Let $h_{i} \rightarrow 0+, r_{i} \rightarrow r$ be such that $D_{+} \varphi\left(x_{0}\right) r=\lim _{i \rightarrow \infty}\left[\varphi\left(x_{0}+h_{i} r_{i}\right)-\varphi\left(x_{0}\right)\right] / h_{i}$. Then

$$
\begin{aligned}
D_{+} \varphi\left(x_{0}\right) r-<p, r> & =\lim _{i \rightarrow \infty}\left[\varphi\left(x_{0}+h_{i} r_{i}\right)-\varphi\left(x_{0}\right)-<p, h_{i} r_{i}>\right] / h_{i} \\
& \geq\|r\| \liminf _{x \rightarrow x_{0}}\left[\varphi(x)-\varphi\left(x_{0}\right)-<p, x-x_{0}>\right] /\left\|x-x_{0}\right\| \geq 0
\end{aligned}
$$

(by definition of the subdifferential). Thus for all $r, D_{+} \varphi\left(x_{0}\right) r \geq\langle p, r\rangle$. 
To prove the equality in (2.15) consider $p$ satisfying:

$$
\forall r \in \mathbf{R}^{m}, D_{+} \varphi\left(x_{0}\right) r \geq\langle p, r>
$$

Let $x_{i} \rightarrow x_{0}$ be such that

$$
\begin{aligned}
& \liminf _{x \rightarrow x_{0}} {\left[\varphi(x)-\varphi\left(x_{0}\right)-<p, x-x_{0}>\right] /\left\|x-x_{0}\right\| } \\
& \quad=\lim _{i \rightarrow \infty}\left[\varphi\left(x_{i}\right)-\varphi\left(x_{0}\right)-<p, x_{i}-x_{0}>\right] /\left\|x_{i}-x_{0}\right\|
\end{aligned}
$$

Taking a subsequence we may assume that $\left(x_{i}-x_{0}\right) /\left\|x_{i}-x_{0}\right\|$ converges to some $r$. Then

$$
\lim _{i \rightarrow \infty}\left[\varphi\left(x_{i}\right)-\varphi\left(x_{0}\right)-<p, x_{i}-x_{0}>\right] /\left\|x_{i}-x_{0}\right\| \geq D_{+} \varphi\left(x_{0}\right) r-<p, r>\geq 0
$$

and therefore $p \in \partial_{-} \varphi\left(x_{0}\right)$. This ends the proof of (2.15). To prove (2.16) observe that $\partial_{+} \varphi\left(x_{0}\right)=-\partial_{-}(-\varphi)\left(x_{0}\right)$ and therefore (2.16) is a consequence of (2.15).

Theorem 2.8. If a function $V: \Omega \rightarrow \mathbf{R}$ verifies relations (2.13), then $V$ is a viscosity solution to Hamilton-Jacobi equation (2.14).

Proof. By (2.15), (2.13) for all $(p, q) \in \partial_{-} V(t, x) \subset \mathbf{R} \times \mathbf{R}^{n}$

$$
p-H(t, x,-q)=\inf _{u \in \operatorname{co} F(t, x)}(p+<q, u>) \leq \inf _{u \in \operatorname{co} F(t, x)} D_{+} V(t, x)(1, u) \leq 0
$$

Thus for all $(p, q) \in \partial_{-} V(t, x)$

$$
-p+H(t, x,-q) \geq 0
$$

On the other hand by $(2.16),(2.13)$ for all $(p, q) \in \partial_{+} V(t, x)$

$$
-p+H(t, x,-q)=\sup _{u \in \operatorname{co} F(t, x)}(-p-<q, u>) \leq \sup _{u \in \operatorname{co} F(t, x)} D_{+}(-V)(t, x)(1, u) \leq 0 .
$$

The very definition of viscosity solution ends the proof.

\section{Solutions of contingent inequalities and optimal trajectories}

Recall that the value function is nondecreasing along the trajectories of differential inclusion (1.1). We show next that every upper semicontinuous solution of inequality (2.7) enjoy the above property.

Theorem 3.1. Let $V:[0,1] \times \mathbf{R}^{n} \rightarrow \mathbf{R} \bigcup\{ \pm \infty\}$ be an upper semicontinuous function satisfying the inequality 


$$
\sup _{u \in c o f(t, x)} D_{+}(-V)(t, x)(1, u) \leq 0,(t, x) \in \operatorname{Dom} V, t<1
$$

If $F$ is locally Lipschitzian in both variables then for every trajectory $x \in S_{[t, 1]}$ satisfying $(s, x(s)) \in \operatorname{Dom} V$, the function $s \rightarrow V(s, x(s))$ is nondecreasing on $[t, 1]$.

Proof. Consider the closed set $K=\operatorname{epi}(-V)$. By $[2, \mathrm{p} .418]$ epi $D_{+}(-V)(s, x)$ is equal to the contingent cone $T_{K}(s, x,-V(s, x)$, and by (3.1) for all $(s, x) \in \operatorname{dom} V, s \in[0,1[, q \geq-V(s, x)$

$$
(1, F(s, x), 0) \subset T_{K}(s, x,-V(s, x)) \subset T_{K}(s, x, q)
$$

Fix a trajectory $x \in S_{[t, 1]}, t \in[0,1]$, such that $(s, x(s)) \in \operatorname{Dom} V$ and consider the function $g:[t, 1] \rightarrow \mathbf{R}_{+}$defined by

$$
g(s)=\operatorname{dist}_{K}(s, x(s),-V(t, x(t)))
$$

Observe that $g(t)=0$.

Step 1. We claim that $g \equiv 0$ on $[t, 1]$. Indeed assume for a while that for some $T \in[t, 1], g(T)>0$. For all $s \in[t, 1]$, let $\pi(s) \in K$ be such that

$$
g(s)=\|(s, x(s),-V(t, x(t)))-\pi(s)\|
$$

By continuity of $g$ there exist $t \leq t_{0}<t_{1}<T$ such that $g\left(t_{0}\right)=0, g>0$ on $\left.] t_{0}, t_{1}\right]$ and for all $\left.s \in] t_{0}, t_{1}\right]$

$$
\pi(s)=(\bar{s}, \bar{y}, q) \text { for some } \bar{s} \in[0,1][,\|\bar{y}-x(s)\| \leq 1, q \geq-V(\bar{s}, \bar{y}) .
$$

To end the proof of Step 1 we verify that $g=0$ on $\left[t_{0}, t_{1}\right]$. Indeed $g$ being a Lipschitzian function, by Gronwall's inequality, it is enough to show that for a constant $L>0$

$$
g(s) \leq L g(s) \text { a. e. in }\left[t_{0}, t_{1}\right]
$$

Let $L$ be the Lipschitz constant of $F$ on the set $\{(s, x(s)+B): s \in[t, 1]\}$. By the Rademacher theorem $g$ is differentiable almost everywhere. Let $s \in\left[t_{0}, t_{1}\right]$ be a point where the derivatives $g_{\prime}(s)$ and $x \prime(s) \in F(s, x(s))$ do exist. Since $x(s+h)=x(s)+h x \prime(s)+0(h)$, applying the inequality of $[1, \mathrm{p} .202]$ we obtain that $\left.g_{\prime}^{\prime}(s) \leq \operatorname{dist}((1, x)(s), 0), T_{K}(\pi(s))\right)$. Thus, by (3.4), (3.2) for some $\bar{s} \in[0,1[, \bar{y} \in x(s)+B$

$$
\left.g^{\prime}(s) \leq \operatorname{dist}\left(\left(1, x^{\prime}(s), 0\right),(1, F(\bar{s}, \bar{y}), 0)\right) \leq L\left(\|\bar{s}-s\|^{2}+\| x(s)-\bar{y}\right) \|^{2}\right)^{1 / 2} \leq L g(s)
$$

and (3.5) follows. 
Step 2. By Step $1, \operatorname{dist}_{K}(s, x(s),-V(t, x(t)))=0$ on $[t, 1]$ and thus for all $s \in[t, 1],-V(s, x(s)) \leq-V(t, x(t))$. Hence for every $t \leq s \leq 1, V(s, x(s) \geq V(t, x(t))$. Since $t \in[0,1]$ is arbitrary the proof is complete.

Recall that the value function is constant along optimal trajectories.

We provide next sufficient conditions for the solution $V$ to inequalities (2.6), (2.7) to be constant along at least one trajectory.

From now until the end of the section we assume that for some $a>0$ and all $t \in[0,1], x \in \mathbf{R}^{n}$

$$
\sup \{|u|: u \in F(t, x)\} \leq a(|x|+1)
$$

Theorem 3.2. Let $P:[0,1] \rightrightarrows \mathbf{R}^{n}$ be a set valued map with nonempty images and closed graph. Assume that for all $x \in P(t), t \in[0,1], F(t, x)$ is convex. Let $V: \operatorname{graph} P \rightarrow \mathbf{R}$ be a continuous function satisfying the inequality:

for all $t \in\left[0,1\left[, x \in P(t)\right.\right.$ there $\exists u \in F(t, x)$ such that $D_{+} V(t, x)(1, u) \leq 0$.

Then for all $(t, \xi) \in$ graph $P$ there exists $z \in S_{[t, 1]}(\xi)$ such that the function $s \rightarrow V(s, z(s))$ is nonincreasing on $[t, 1]$.

Corollary 3.3. Let $V:[0,1] \times \mathbf{R}^{n} \rightarrow \mathbf{R}$ be a continuous function satisfying inequalities

$$
\begin{aligned}
& \sup _{u \in c o F(t, x)} D_{+}(-V)(t, x)(1, u) \leq 0,(t, x) \in\left[0,1\left[\times \mathbf{R}^{n}\right.\right. \\
& \inf _{u \in c o F(t, x)} D_{+} V_{d}(t, x)(1, u) \leq 0,(t, x) \in\left[0,1\left[\times \mathbf{R}^{n}\right.\right.
\end{aligned}
$$

and assume that $F$ has convex images and is locally Lipschitzian. Then for every $(t, \xi) \in[0,1] \times \mathbf{R}^{n}$ there exists $z \in S_{[t, 1]}(\xi)$ such that $V(s, z(s))=$ const on $[t, 1]$.

Proof. By (3.7) for every $(t, x) \in \mathrm{graph} P, t<1$ there exists $v \in 1 \times F(t, x)$ such that $D_{+} V(t, x) v \leq 0$. By the poof of $\left[1\right.$, pp. 296-298] for every $\left(t, y_{0}\right) \in \operatorname{graph} P, t<1$, there exists $T>0$ and a trajectory $y:\left\{0, T\left[\rightarrow \mathbf{R}^{n}\right.\right.$ of the differential inclusion

$$
y^{\prime}(s) \in F(t+s, y(s)), y(0)=y_{0}
$$

such that the function $s \rightarrow V(t+s, y(s))$ is nonincreasing on $[0, T$. Set $x(t+s)=y(s)$. Then $x \in S_{[t, T \mid}\left(y_{0}\right)$ and since $F$ satisfies (3.6) the derivative $x$ is essentially bounded and therefore $x$ can be extended on the time interval $[t, T]$. Moreover by the continuity of $V$, for all $s \in[t, T], V(T, x(T)) \leq V(s, x(s))$ and thus $V(t, x(t))$ is nonincreasing on $[t, T]$. Since $\left(t, y_{0}\right) \in\left[0,1\left[\times \mathbf{R}^{n}\right.\right.$ is arbitrary for every $(t, \xi) \in\left[0,1\left[\times \mathbf{R}^{n}\right.\right.$ there exist $t<\ldots<t_{i}<t_{i+1} \cdots<1$ and $x_{1} \in S_{\left[t, t_{1}\right]}(\xi), \quad x_{i+1} \in S_{\left[t_{i}, t_{i+1}\right]}\left(x_{i}\left(t_{i}\right)\right)$ such that $t \rightarrow V\left(t, x_{i}(t)\right)$ is nonincreasing on 
$\left[t_{i-1}, t_{i}\right] . \quad$ By (3.6) we may assume that $t_{i} \rightarrow 1$. Setting $z(t)=x_{i}(t)$ for $t \in\left[t_{i-1}, t_{i}\right], z(1)=\lim _{i \rightarrow \infty} x_{i}\left(t_{i}\right)$ we end the proof.

From Section 2 we know that the value function verifies inequalities (3.1), (3.7) with graph $P=D o m V$ and the boundary condition

$$
V(1, x)=g(x), x \in \mathbf{R}^{n}
$$

Actually we have

Theorem 3.4. Assume that the domain of definition of the value function $V$ is closed and equal to $\{(t, P(t)): t \in[0,1]\}$, where $P(t) \neq \varnothing$ for all $t$, and $F$ has convex images on graph $P$. If a continuous function $W: \operatorname{graph} P \rightarrow \mathbf{R}$ satisfies (3.6), (3.8) then $V \leq W$.

Proof. Fix a continuous solution $W$ of (3.6), (3.8) and let $(t, \xi) \in \mathrm{graph} P$. By Theorem 3.2 there exists $z \in S_{[t, 1]}(\xi)$ such that $s \rightarrow W(s, z(s))$ is nonincreasing on $[t, 1]$. Thus

$$
V(t, \xi)=\inf \left\{g(x(1)): x \in S_{[t, 1]}(\xi)\right\} \leq g(z(1))=W(1, z(1)) \leq W(t, \xi)
$$

and the result follows.

Theorems 3.1, 3.2 and results of Section 2 imply

Corollary 3.5. Let $V:[0,1] \times \mathbf{R}^{n} \rightarrow \mathbf{R}$ be as in Corollary 3.3 and assume that $F$ is locally Lipschitzian and has convex images. Then there exists an optimal solution $z$ to problem (2.1) such that $V(t, z(t))=$ const on $[0,1]$.

\section{Optimal feedback}

Observe that if $V$ and $z$ are as in Theorem 3.2 then for all $s \in[t, 1[$ and small $h>0, V(s+h, z(s+h)) \leq V(s, z(s))$. Thus $D_{+} V(s, z(s))(1, z(s)) \leq 0$ whenever the derivative $z(s)$ do exist. For all $(s, x) \in \mathbf{R} \times \mathbf{R}^{n}$ set

$$
G(s, x)=\left\{u \in F(s, x): D_{+} V(s, x)(1, u) \leq 0\right\}
$$

and consider the differential inclusion

$$
x^{\prime} \in G(s, x)
$$

Hence $z$ is a trajectory of (4.1). Corollary 3.5 implies then 
Theorem 4.1. Let $V:[0,1] \times \mathbf{R}^{n} \rightarrow \mathbf{R}$ be a continuous solution to the boundary problem

$$
\left.\begin{array}{l}
\sup _{u \in \operatorname{co} F(t, x)} D_{+}(-V)(t, x)(1, u) \leq 0,(t, x) \in\left[0,1\left[\times \mathbf{R}^{n}\right.\right. \\
\left.\inf _{u \in \operatorname{co} F(t, x)} D_{+} V(t, x)(1, u) \leq 0,(t, x)\right) \in\left[0,1\left[\times \mathbf{R}^{n}\right.\right. \\
V(1, x)=g(x) \text { on } \mathbf{R}^{n}
\end{array}\right\}
$$

and assume that $F$ is locally Lipschitzian,satisfies (3.6), and has convex images. Then there exists a trajectory $z \in W^{1,1}(0,1)$ of the differential inclusion (4.1) which is an optimal trajectory of problem (2.1).

Observe that the set-valued map $G$ has compact images. If we assume more regularity on $V$ or $G$ then every solution of (4.1) is an optimal trajectory of the problem (2.1).

Theorem 4.2. Let $V:[0,1] \times \mathbf{R}^{n} \rightarrow \mathbf{R}$ be a locally Lipschitzian solution of the boundary problem (4.2) and assume that $F$ is locally Lipschitzian. Then every trajectory of the inclusion (4.1) defined on the time interval $[0,1]$ is optimal.

Proof. Let $z$ be a trajectory of (4.1). The function $\varphi(t)=V(t, z(t))$ is locally Lipschitzian. By the Rademacher theorem it is differentiable almost everywhere.

Let $t$ be so that $z(t)$ do exist and $h_{i} \rightarrow 0+$ such that

$$
D_{+} V(t, z(t))(1, z(t))=\lim _{i \rightarrow 0+} \frac{V\left(t+h_{i}, z\left(t+h_{i}\right)\right)-V(t, z(t))}{h_{i}}
$$

Thus $\varphi^{\prime}(t)=D_{+} V(t, z(t))\left(1, z^{\prime}(t)\right) \leq 0$ and $\varphi$ is nonincreasing. By Theorem $3.1 \varphi$ is also nondecreasing. Thus $\varphi=$ const. Proposition 2.1 ends the proof.

Another sufficient condition for the trajectories of (4.1) to be optimal requires more regularity of $G$ and less of $V$.

Theorem 4.3. Let $V:[0,1] \times \mathbf{R}^{n} \rightarrow \mathbf{R}$ be a continuous solution of boundary problem (4.2) and assume that $G$ has compact convex images and is locally Lipschitzian. Then every $W^{1,1}(0,1)$-trajectory of the inclusion (4.1) is an optimal trajectory for the problem (2.1).

Proof. By definition of $G$ for all $t \in\left[0,1\left[, x \in \mathbf{R}^{n}\right.\right.$

$$
\sup _{u \in G(t, x)} D_{+}-(-V)(t, x)(1, u) \leq 0
$$

Thus, by Theorem 3.1, for every trajectory $z$ of the inclusion (4.1) the function $s \rightarrow-V(s, z(s))$ is nondecreasing and by Theorem 3.1 the function $s \rightarrow V(s, z(s))$ is nondecreasing. Thus $V(s, z(s))=$ const and by Proposition 2.1, $z$ is an optimal solution of the problem (2.1). 


\section{Other relations satisfied by a locally Lipschitzian value function}

Using Theorem 1.1 one can easily check that whenever the value function is locally Lipschitzian in $x$ then it also verifies the relations

$$
\begin{aligned}
& \left.\left.\sup _{u \in \operatorname{co} F(t, x)} D_{+} V(t, x)(-1,-u) \leq 0,(t, x) \in\right] 0,1\right] \times \mathbf{R}^{n} \\
& \left.\left.\inf _{u \in c o F(t, x)} D_{+}(-V)(t, x)(-1,-u) \geq 0(t, x) \in\right] 0,1\right] \times \mathbf{R}^{n}
\end{aligned}
$$

and for all $(t, x) \in\left[0,1\left[\times \mathbf{R}^{n}\right.\right.$

$$
\inf _{u \in c o F(t, x)} \limsup _{h \rightarrow 0+}[V(t+h, x+h u)-V(t, x)] / h=0
$$

It also verifies two relations involving epiderivative introduced by Clarke [8]:

Definition 5.1. Let $\varphi: \mathbf{R}^{m} \rightarrow \mathbf{R}$ be a locally Lipschitzian function. The epiderivative $\varphi^{0}(x): \mathbf{R}^{m} \rightarrow \mathbf{R}$ is defined by: for all $u \in \mathbf{R}^{m}$.

$$
\varphi^{0}(x) u=\lim _{\substack{x^{\prime} \rightarrow x \\ h \rightarrow 0+}} \sup _{\substack{x \\ x^{\prime}}}\left[\varphi(h u)-\varphi\left(x^{\prime}\right)\right] / h
$$

The generalized gradient $\partial \varphi(x)$ is given by

$$
\partial \varphi(x)=\left\{p \in \mathbf{R}^{m}: \forall u \in \mathbf{R}^{m},\left\langle p, u>\leq \varphi^{0}(x) u\right\}\right.
$$

Observe that $\varphi^{0}(x) \geq D_{+} \varphi(x)$ and therefore $\partial_{-} \varphi(x) \subset \partial \varphi(x)$, $\partial_{+}(-\varphi)(x) \subset-\partial \varphi(x)=\partial(-\varphi)(x)$.

For all $(t, x) \in] 0,1\left[\times \mathbf{R}^{n}\right.$ set

$$
\begin{aligned}
& \pi_{t} \partial V(t, x)=\{p: \exists q \text { such that }(p, q) \in \partial V(t, x)\} \\
& \pi_{x} \partial V(t, x)=\{q: \exists p \text { such that }(p, q) \in \partial V(t, x)\}
\end{aligned}
$$

i.e. $\pi_{t} \partial V(t, x)$ and $\pi_{x} \partial V(t, x)$ are projections of $\partial V(t, x)$ on $t$ and $x$ spaces respectively.

Theorem 5.2 Assume that the value function is locally Lipschitzian on $] 0,1\left[\times R^{n}\right.$. Then

$$
\min _{(p, q) \in \partial V(t, x)}(p-H(t, x,-q))=0
$$

and

$$
\max _{\pi_{t} \partial V(t, x)} p=\max _{q \in \pi_{x} \partial V(t, x)} H(t, x,-q)
$$

Moreover for all $(\bar{p}, \bar{q}) \in \partial V(t, x)$ satisfying 


$$
\max _{q \in \pi_{x} \partial V(t, x)} H(t, x,-q)=H(t, x,-\bar{q})
$$

we have $\bar{p}=\max _{\pi_{t} \partial V(t, x)} p$

Corollary 5.3. If $V$ is a locally Lipschitzian solution of (5.1) then $V$ is a viscosity supersolution of the equation

$$
\frac{\partial V}{\partial t}-H\left(t, x,-\frac{\partial}{\partial x} V\right)=0
$$

and a viscosity subsolution of the equation

$$
-\frac{\partial}{\partial t} V+H\left(t, x,-\frac{\partial}{\partial x} V\right)=0
$$

Remark. The extension of the Hamilton-Jacobi equation to the form (5.1) was first introduced in Offin [21] (see Clarke-Vinter [6], Clarke [8]).

Broof. By (3.2) for all $(t, x) \in] 0,1\left[\times \mathbf{R}^{n}(1, F(t, x), 0)\right.$ belongs to the contingent cone to epi $(-V)$ at $(t, x,-V(t, x))$. Thus, by $[2$, p. 409] and continuity of $F$ for all $(t, x) \in] 0,1\left[\times \mathbf{R}^{n},(1, F(t, x), 0)\right.$ belong to the tangent cone of Clarke to epi $(-V)$ at $(t, x,-V(t, x))$.

This implies that for all $u \in F(t, x),(-V)^{0}(t, x)(1, u) \leq 0$ (see [2, p. 421]). Using the equality $\partial V(t, x)=-\partial(-V)(t, x)$ we finally obtain

$$
\sup _{(p, q) \in \partial V(t, x)} \sup _{u \in c o f(t, x)}<-p-q, u>\leq 0
$$

Since $\partial V(t, x)$ and $c o F(t, x)$ are compact from (5.3) follows that

$$
\min _{(p, q) \in \partial V(t, x)}\left(p+\min _{u \in F(t, x)}<q, u>\right) \geq 0
$$

To prove the equality in (5.4) we have to verify that for some $\bar{u} \in \operatorname{co} F(t, x),(-V)^{0}(t, x)(1, \bar{u}) \geq 0$. By Theorem 1.1 there exist $w_{h} \in R(t+h, t) x$ such that $\operatorname{dist}\left(w_{h}, F(t, x)\right) \rightarrow 0$ when $h \rightarrow 0+$ and $\| V(t, x)-V\left(t+h, w_{h} \|=0(h)\right.$.

Let $w_{i}=w_{h_{i}}, h_{i} \rightarrow 0+$ be a subsequence such that $\left(w_{i}-x\right) / h_{i}$ converge to some $\bar{u} \in \operatorname{co} F(t, x)$. Then $0=\lim _{i \rightarrow \infty}\left[V(t, x)-V\left(t+h_{i}, w_{i}\right)\right] / h_{i} \leq(-V)^{0}(t, x)(1, \bar{u})$. Therefore we have an equality in (5.4) and (5.1) follows. To prove (5.2) fix $u \in F(t, x)$. By Theorem 1.1 for all $\left(t^{\prime}, x^{\prime}\right)$ near $(t, x)$ and small $h>0$

$$
V\left(t^{\prime}+h, x^{\prime}\right)-V\left(t^{\prime}+h, x^{\prime}+h u\right) \leq V\left(t^{\prime}+h, x^{\prime}\right)-V\left(t^{\prime}, x^{\prime}\right)+0\left(\dot{t}^{\prime}, x^{\prime}, h\right)
$$

Thus $(-V)^{0}(t, x)(0, u) \leq V^{0}(t, x)(1,0)$. 
Since $u \in F(t, x)$ is arbitrary we proved that

$$
\max _{u \in F(t, x)}(-V)^{0}(t, x)(0, u) \leq V^{0}(t, x)(1,0)
$$

To prove the opposite inequality fix $\left(t_{i}, x_{i}\right) \rightarrow(t, x)$ and $h_{i} \rightarrow 0+$ such that

$$
V^{0}(t, x)(1,0)=\lim _{i \rightarrow \infty}\left[V\left(t_{i}+h_{i}, x_{i}\right)-V\left(t_{i}, x_{i}\right)\right] / h_{i}
$$

Let $w_{i} \in R\left(t_{i}+h_{i}, t_{i}\right) x_{i}$ be such that $\left\|V\left(t_{i}, x_{i}\right)-V\left(t_{i}+h_{i}, w_{i}\right)\right\|=0\left(h_{i}\right)$. By Theorem 1.1 there exist $u_{i} \in \operatorname{co} F(t, x)$ such that $\left\|w_{i}-x_{i}-h_{i} u_{i}\right\|=0\left(h_{i}\right)$. Taking if needed a subsequence we may assume that $\lim _{i \rightarrow \infty} u_{i}=\bar{u} \in F(t, x)$. Then, by (5.5),

$$
\begin{aligned}
V^{0}(t, x)(1,0) & =\lim _{i \rightarrow \infty}\left[V\left(t_{i}+h_{i}, x_{i}\right)-V\left(t_{i}+h_{i}, x_{i}+h_{i} u_{i}\right)\right] / h_{i} \\
& \leq(-V)^{0}(t, x)(0, \bar{u}) \leq \max _{u \in c o F(t, x)}(-V)^{0}(t, x)(0, u)
\end{aligned}
$$

Since $(-V)^{0}(t, x)$ is a convex continuous function the maximum is attained at an extremal of $\operatorname{co} F(t, x)$. Thus

$$
V^{0}(t, x)(1,0)=\max _{u \in F(t, x)}(-V)^{0}(t, x)(0, u)
$$

Using again that $\partial V(t, x)=-\partial(-V)(t, x)$ we derive from (5.6) that

$$
\max _{\pi_{t} \partial V(t, x)} p=\max _{q \in \pi_{x} \partial V(t, x) u \in F(t, x)} \max _{u-q, u>}<
$$

Hence (5.2). To prove the last statement fix $(\bar{p}, \bar{q}) \in \partial V(t, x)$ such that $\min _{q \in \pi_{x} \partial V(t, x)} \min _{u \in F(t, x)}\langle q, u\rangle=\min _{u \in F(t, x)}\langle\bar{q}, u\rangle$. Then, by $(5.2), \bar{p} \leq H(t, x,-\bar{q})$ and the result follows from (5.1).

\section{Superdifferentials of the value function - Pontrjagin's principle}

In this section we relate the adjoint solution of the Pontrjagin maximum principle to the superdifferential of the value function.

Our basic tools are the results of $[13],[16]$.

Let $f: \mathbf{R}^{n} \times \mathbf{R}^{m} \rightarrow \mathbf{R}^{n}$ be a locally Lipschitzian differentiable function and $U: \mathbf{R}^{n} \rightarrow \mathbf{R}^{m}$ be a locally Lipschitzian set-valued map with compact images. Consider the problem 


$$
\text { minimize } g(x(1))
$$

over the solutions of the closed loop control system

$$
\begin{gathered}
x=f(x, u), u \in U(x), \\
x(0) \in K
\end{gathered}
$$

where $g$ is a differentiable function and $K$ is a given set of initial states. We recall that the contingent cone to $K$ at $x \in K$ is given by

$$
T_{K}(x)=\left\{v: \lim \inf _{h \rightarrow 0+} \operatorname{dist}\left(v, \frac{K-x}{h}\right)=0\right\}
$$

Let $V$ be the value unction associated to the problem, i.e.

$$
V(t, \xi)=\inf \left\{g(x(1)): x \in W^{1,1}(t, 1) \text { is a solution of }(5.2), x(t)=\xi\right\}
$$

By the Theorem of Appendix, $V$ is a locally Lipschitzian function.

Theorem 6.1. Assume that a trajectory control pair $\left(z, u_{*}\right)$ solves the above problem and that there exist linear operators $B(s) \in \mathbf{L}\left(\mathbf{R}^{n}, \mathbf{R}^{n}\right), s \in[0,1]$ satisfying

i) for all $x \in \mathbf{R}^{n}, s \rightarrow B(s) x$ is measurable

ii) for some $k \in L^{\infty}(0,1)$ and almost all $s,\|B(s)\| \leq k(s)$

iii) for almost all $s$ and all $x \in \mathbf{R}^{n}$

$$
\lim _{h \rightarrow 0+} \operatorname{dist}\left(B(s) x, \frac{U(z(s)+h u)-u *(s)}{h}\right)=0
$$

Then there exists a function $q \in W^{1, \infty}(0,1)$ satisfying

$$
\begin{gathered}
-q^{\prime}(t)=\left(\frac{\partial}{\partial x} f(z(t), u *(t))^{*}+B(t)^{*} \frac{\partial}{\partial u} f(z(t), u *(t))\right)^{*} q(t) \text { a.e. in }[0,1] \\
<q(t), z^{\prime}(t)>=\max _{u \in U(z(t))}<q(t), f(z(t), u)>\text { a.e. in }[0,1] \\
q(0) \in T_{K}(z(0))^{-}=\left\{p: \forall u \in T_{K}(0),<p, u>\leq 0\right\} \\
-q(1)=g^{\prime}(z(1)) \\
-q(t) \in \partial_{+}^{x} V(t, z(t)) \text { for all } t \in[0,1]
\end{gathered}
$$

where $\partial_{+}^{x} V$ denote the superdifferential of $V$ with respect to $x$. Moreover for all $t$ such that the derivative $z(t)$ exists

$$
(H(z(t), q(t)),-q(t)) \in \partial_{+} V(t, z(t)) .
$$


Remark. The last statement of the above theorem and Theorem 2.8 imply that for almost all $t \in[0,1]$ there exist $(\alpha, \beta) \in \partial_{+} V(t, z(t))$ such that

$$
\alpha+H(t, z,-\beta)=0
$$

Remark. Assumptions i) - iii) are in particular verified when there exists a selection $p(t, x) \in U(x), t \in[0,1], x \in \mathbf{R}^{n}$ measurable in $t$ such that for all $t, p(t, z(t))=u *(t)$ and $p(t, \cdot)$ is differentiable at $z(t)$. Then we can take for $B(t)$ the matrix $\frac{\partial p}{\partial x}(t, z(t)$ ) (compare Leitmann [18]).

Proof. For all $x \in \mathbf{R}^{n}$, set

$$
F(x)=\{f(x, u): u \in U(x)\}
$$

By the example of Section 1 we can replace the dynamical system (5.2) by the differential inclusion $x \in F(x)$.

From [13] follows the existence of a function $q \in W^{1, \infty}(0,1)$ verifying (6.4)-(6.7).

Consider the linear equation

$$
\dot{w}=\left(\frac{\partial f}{\partial x}(z(s), u *(s))+\frac{\partial f}{\partial u}(z(s), u *(s)) B(s)\right) w
$$

and let $w \in W^{1,1}(0,1)$ be a trajectory of (6.9). By [15] there exist trajectories $x_{h} \in W^{1,1}(t, 1)$ of (6.2) satisfying $\lim _{h \rightarrow 0+}\left(x_{h}-z\right) / h=w$ (in $W^{1,1}(t, 1)$ ). Thus, by (6.7), (6.4), for all $w(t) \in \mathbf{R}^{n}$

$$
\begin{aligned}
<q(t), w(t)> & =-g^{\prime}(z(1)) w(1) \leq \lim \inf _{h \rightarrow 0+}[V(t, z(t))-V(t, z(t)+h w(t))] / h \\
& =D_{+}^{x}(-V)(t, z(t)) w(t)
\end{aligned}
$$

and (6.8) follows from (2.16). Let $t \in[0,1]$ be such that the derivative $z(t)$ does exist. Then for all trajectory $w \in W^{1,1}(0,1)$ of (6.9) and $\alpha \in \mathbf{R}$

$$
\begin{aligned}
& -D_{+}(-V)(t, z(t))\left(\alpha, \alpha z^{\prime}(t)+w(t)\right) \\
& =\lim \sup _{h \rightarrow 0_{+}}\left[V\left(t+\alpha h, z(t)+h\left(\alpha z^{\prime}(t)+w(t)\right)\right)-V(t, z(t))\right] / h \\
& =\lim \sup _{h \rightarrow 0_{+}}[V(t+\alpha h, z(t+\alpha h)+h w(t+\alpha h))-V(t, z(t))] / h \\
& \leq g^{\prime}(z(1)) w(1)=<-q(t), w(t)>=<-q(t),-\alpha z^{\prime}(t)>+<-q(t), \alpha z^{\prime}(t)+w(t)>
\end{aligned}
$$


Hence, using (6.5) we obtain that for all $(\alpha, u) \in \mathbf{R} \times \mathbf{R}^{n}$

$$
D_{+}(-V)(t, z(t))(\alpha, u) \geq-\alpha H(z(t), q(t))+<q(t), u>
$$

Hence $(H(z(t), q(t)),-q(t)) \in \partial_{+} V(t, z(t))$. The proof is complete. $\square$.

\section{Appendix. Regularity of the value function}

Theorem 1. Assume that $F$ satisfies $\left(H_{1}\right)-\left(H_{3}\right)$ and (3.6).

i) If $g$ is lower-semicontinuous and if $F$ has convex images then $V$ is also lower semicontinuous.

Assume next that $g$ is bounded from below.

ii) If the domain of definition of $g$ is closed and $F$ has convex images then $V$ has a closed domain of definition

iii) If the domain of definition of $g$ is open and $g$ is uniformly continuous on it then $V$ has an open domain of definition and is continuous on it

iv) If $g$ is Lipschitzian on its open domain of definition then $V$ is locally Lipschitzian on its open domain of definition.

Proof. Let $\left(t_{i}, \xi_{i}\right) \in \operatorname{Dom} V$ be a sequence converging to some $(t, \xi)$ and $x_{i} \in S_{\left[t_{i}, 1\right]}\left(\xi_{i}\right)$ satisfy

$$
V\left(t_{i}, \xi_{i}\right) \leq g\left(x_{i}(1)\right) \leq V\left(t_{i}, \xi_{i}\right)+1 / i
$$

Let $t_{0}=\inf t_{i}$. By $\left[1\right.$, p. 120] we can extend every trajectory on the time interval $\left[t_{0}, 1\right]$ and by $\left[1\right.$, p. 104] there exists a subsequence $x_{i_{k}}$ converging uniformly to a trajectory $x \in S_{\left[t_{0}, 1\right]}(\xi)$. Thus

$$
V(t, \xi) \leq g(x(1)) \leq \lim \inf _{k \rightarrow \infty} g\left(x_{i_{k}}(1)\right)=\lim \inf _{i \rightarrow \infty} V\left(t_{i}, \xi_{i}\right)
$$

and we proved i). If $g$ is bounded from below then $V$ is also bounded from below. The last inequality implies also ii). To prove iii) fix $(t, \xi) \in D o m ~ V$. By $[1$, p. 120] for every $\epsilon>0$ there exists $\delta>0$ such that $\left\|\left(t^{\prime}, \xi^{\prime}\right)-(t, \xi)\right\|<\delta$ implies that the Hausdorff distance $d_{H}\left(R(1-t, t) \xi, R\left(1-t^{\prime}, t^{\prime}\right) \xi^{\prime}\right)<\epsilon$. Fix any $\rho>0$ and let $\epsilon>0$ be so that $\left\|y-y_{1}\right\|<\epsilon$ implies that $\left\|g(y)-g\left(y_{1}\right)\right\|<\rho$. Let $\delta>0$ be as above. If $\left\|\left(t^{\prime}, \xi^{\prime}\right)-(t, \xi)\right\|<\delta$ then for every $x \in S_{[t, 1]}(\xi)$, there exists $y \in S_{\left[t^{\prime}, 1\right]}\left(\xi^{\prime}\right)$ such that $g(y(1)) \leq g(x(1))+\rho$ and for all $y \in S_{\left[t^{\prime}, 1\right]}\left(\xi^{\prime}\right)$ there exists $x \in S_{[t, 1]}(\xi)$ satisfying $g(x(1)) \leq g(y(1))+\rho$. This implies iii). To prove iv) we show first that $V$ is locally Lipschitzian in $\xi$. From $[1$, p. 120] we know that the map $(t, \xi) \rightarrow S_{[t, 1]}(\xi)$ is locally Lipschitzian in $\xi$ with the constant independent of $t$. Since $g$ is 
Lipschitzian on dom $g$ we obtain that $V$ is locally Lipschitzian in $\xi$. Fix $\left(t_{0}, \xi_{0}\right) \in[0,1] \times \mathbf{R}^{n}$ and let $\mathbf{N}$ be a neighborhood of $\left(t_{0}, \xi_{0}\right)$ where $V$ is Lipschitzian in $\xi$ with a constant $M_{1}$. Then for all $t \leq t_{1},(t, \xi),\left(t_{1}, \xi_{1}\right) \in \mathrm{N}$ satisfying $R\left(t_{1}-t, t\right) \xi \in \mathrm{N}$ and for every $x \in S_{[t, 1]}(\xi)$

$$
\begin{aligned}
& \left\|V(t, \xi)-V\left(t_{1}, \xi_{1}\right)\right\| \leq \| V(t, \xi)-V\left(t_{1}, x\left(t_{1}\right)\|+\| V\left(t_{1}, x\left(t_{1}\right)\right)-V\left(t_{1}, \xi_{1}\right) \|\right. \\
& \leq\left\|V(t, \xi)-V\left(t_{1}, x\left(t_{1}\right)\right)\right\|+M_{1}\left\|x\left(t_{1}\right)-\xi_{1}\right\|
\end{aligned}
$$

Since $x\left(t_{1}\right)=\xi+\int_{t}^{t_{1}} x^{\prime}(s) d s$ and $x^{\prime}$ is bounded by a constant $M$ we have $\left\|x\left(t_{1}\right)-\xi_{1}\right\| \leq\left\|\xi-\xi_{1}\right\|+M\left(t_{1}-t\right)$. Since $\inf _{x \in S_{\{t, 1\}}(\xi)}\left\|V(t, \xi)-V\left(t_{1}, x\left(t_{1}\right)\right)\right\|=0$ the proof is complete. $\square$.

\section{References}

[1] Aubin, J.-P. and A. Cellina (1984). Differential Inclusions. Springer-Verlag.

[2] Aubin, J.-P. and I. Ekeland (1984). Applied Nonlinear Analysis. WileyInterscience.

[3] Bellman, R.E. (1957). Dynamic Programming. Princeton University Press.

[4] Bellman, R. and S. Dreyfus (1962). Applied Dynamic Programming. Princeton University Press.

[5] Cesari, L. (1983). Optimization Theory and Applications. Springer-Verlag.

[6] Clarke, F.H. and R.B. Vinter (1983). Local optimality conditions and Lipschitzian solutions to the Hamilton-Jacobi equation, SLAM J. of Control and Optimization, 21(6), pp. 856-870.

[7] Clarke, F.H. and R.B. Vinter (1986). On the relationship between the dynamic programming and the maximum principle, Preprint CRM, Universite de Montreal.

[8] Clarke, F.H. (1983). Optimization and Nonsmooth Analysis. Wiley- Interscience.

[9] Crandall, M.G. and P.L. Lions (1983). Viscosity solutions of Hamilton-Jacobi equations, Trans. Amer. Math. Soc., 277, pp. 1-42.

[10] Crandall, M.G., L.C. Evans and P.L. Lions (1984). Some properties of viscosity solutions of Hamilton-Jacobi equation, Trans. Amer. Math. Soc., 282(2), pp. 487502. 
[11] Dreyfus, S.E. (1965). Dynamic Programming and the Calculus of Variations. Academic Press.

[12] Fleming, W.H. and R.W. Rishel (1975). Deterministic and Stochastic Optimal Control. Springer-Verlag.

[13] Frankowska, H. (1986). Contingent cones to reachable sets of control systems, Preprint CRM-1381, University of Montreal.

[14] Frankowska, H. (1986). Local controllability of control systems with feedback, J. Opt. Th. and Appl. (in press).

[15] Frankowska, H. (1987). Local controllability and infinitesimal generators of semigroups of set-valued maps, SIAM J. of Control and Optimization, (25), pp. 412-432.

[16] Frankowska, H. (1987). The maximum principle for the differential inclusion with end point constraints, SIAM J. of Control and Optimization, (25), pp. 145-157.

[17] Jacobson, D. and D. Mayne (1970). Differential Dynamic Programming, Modern Analytic and Computational Methods in Science and Mathematics. American Elsevier Publishing Company.

[18] Leitmann, G. (1982). Optimality and reachability with feedback control, in Dynamical Systems and Microphysics, Academic Press, pp. 119-141.

[19] Lions, P.L. (1982). Generalized solutions of Hamilton-Jacobi Equations, Pitman, Boston.

[20] Lions, P.L., P.E. Souganidis (1985). Differential games, optimal control and directional derivatives of viscosity solutions of Bellman's and Isaaks' Equations, SIAM J. of Control and Optimization, Vol. 23, no. 4.

[21] Offin, D.C. (1978). A Hamilton-Jacobi approach to the differential inclusion problem, Master's thesis, University of British Columbia, Vancouver.

[22] Zeidler, E. (1984). Nonlinear Functional Analysis and its Applications. Vol. III, Springer-Verlag. 